Supplementary Information

SPACEPro: A Software Tool for Analysis of Protein Sample Cleavage for Tandem Mass Spectrometry

Vidur Kailash, Luis Mendoza, Robert L. Moritz\$, and Michael R. Hoopmann\$,*

Institute for Systems Biology, Seattle, WA 98109, USA

\$Equal Senior authors

"Corresponding author:

Michael R. Hoopmann (michael.hoopmann@isbscience.org) 


\section{Supplementary Table of Contents:}

Methods S1: page S-3

Table S1, SPACEPro parameters: page S-4

Figure S1, SPACEPro output: page S-5

Figure S2, SPACEPro JSON output: page S-6

Material S1, Command-line Tutorial: page S-7

Material S2, Trans-Proteomic Pipeline Tutorial: pages S-8 to S-10 
Supplementary Methods 1:

Equations describing the computation of percentage of mis-cleaved, non-specific, and enzymatic peptide abundance for each protein is as follows:

$$
\text { (1) } M C_{p}=\frac{\sum j_{p}}{\sum i_{p}}
$$

where $M C_{p}$ is the percentage of protein $p$ that is mis-cleaved, and $j_{p}$ is the $j$ th mis-cleaved peptide extracted ion chromatogram abundance belonging to protein $p$, and $i$ is the ith peptide extracted ion chromatogram abundance for all peptides belonging to protein $p$.

$$
\text { (2) } \quad N S_{p}=\frac{\sum n_{p}}{\sum i_{p}}
$$

where $N S_{p}$ is the percentage of protein $p$ that is non-specific, and $n_{p}$ is the $n$th mis-cleaved peptide extracted ion chromatogram abundance belonging to protein $p$, and $i$ is the ith peptide extracted ion chromatogram abundance for all peptides belonging to protein $p$.

$$
\text { (3) } E n z_{p}=\frac{\sum z_{p}}{\sum i_{p}}
$$

where $E n z_{p}$ is the percentage of protein $p$ that is enzymatic, and $z_{p}$ is the $z$ th mis-cleaved peptide extracted ion chromatogram abundance belonging to protein $p$, and $i$ is the ith peptide extracted ion chromatogram abundance for all peptides belonging to protein $p$. 
Supplementary Table 1: SPACEPro parameters

\begin{tabular}{|c|c|}
\hline Options & Explanation \\
\hline$-t$, --threshold <number $>$ & PSM probability threshold. Default $=0.9$ \\
\hline$-r$, --rtime $<$ number $>$ & $\begin{array}{l}+/ \text { - retention time (in minutes) to use for precursor } \\
\text { ion extraction. Default }=2.0\end{array}$ \\
\hline$-p,--p p m<$ number $>$ & $\begin{array}{l}+/ \text { - mass error (in parts-per-million) to use for } \\
\text { precursor ion extraction. Default }=10.0\end{array}$ \\
\hline$-\mathrm{c},--$ loc $<$ string $>$ & amino acids where cleavage occurs. Default $=K R$ \\
\hline -a, --anti <string> & $\begin{array}{l}\text { amino acids where cleavage rules are ignored. } \\
\text { Default }=\text { [none] }\end{array}$ \\
\hline$-n,--e n z y$ & $\begin{array}{l}\text { N-terminal sense of enzyme/chemical. If not } \\
\text { specified, sense uses C-terminal as default. }\end{array}$ \\
\hline
\end{tabular}




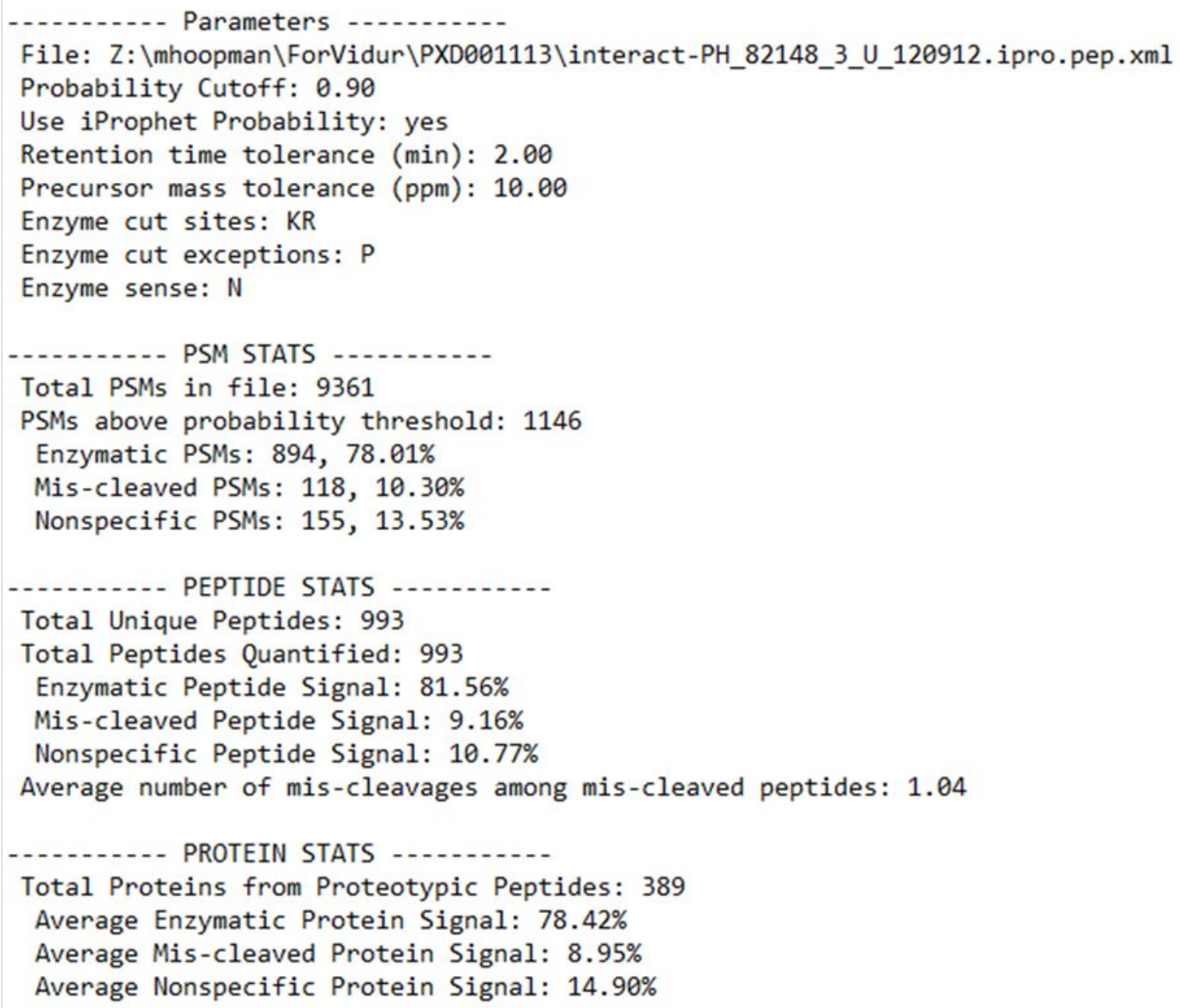

Supplementary Figure 1: Sample output of SPACEPro. 


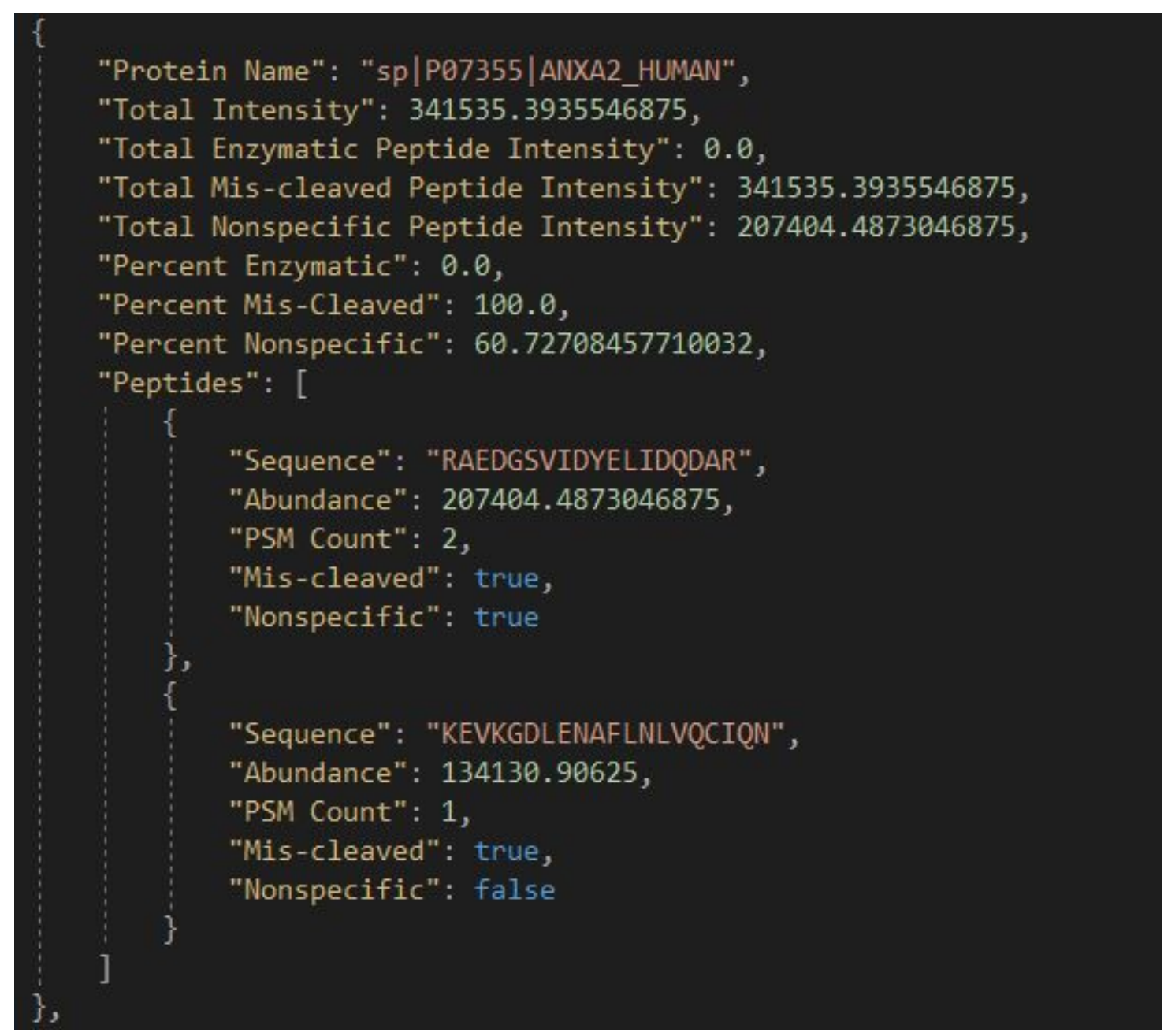

Supplementary Figure 2: Data structure of protein-level SPACEPro results in JSON format. JSON is a human-readable text format consisting of attribute-value pairs. Data pairs can be grouped into objects and stored in arrays. Detailed information can be found at https://www.json.org/json-en.html 


\section{Material S1, Command-line Tutorial:}

SPACEPro can be run on the command with minimal inputs and parameters. For a list of all parameters available to the user, run SPACEPro on the command line without any input file or parameter.

1. Open the command terminal.

2. Navigate to the directory containing database search results in pepXML format.

3. Run the following command:

SPACEPro <input.pep.xml>

Where <input.pep.xml> is the name of the database search results file.

4. Upon completion of SPACEPro, the following files will be generated:

input.pep.xml.ed.txt - A text file containing the summary results of SPACEPro. input.pep.xml.ed.json - A JSON file containing the protein analyses.

5. SPACEPro analysis can be customized with parameters applied after specifying the input pepXML file. For example, to raise the PSM probability threshold to 0.95 , use the following command:

SPACEPro <input.pep.xml> --threshold 0.95

6. It is not necessary to specify the mzML file for precursor ion extraction. The $m z M L$ file is located from elements inside the input pepXML file. However, should the files have been moved to alternate paths in the file system, it is possible to run SPACEPro by placing both the input pepXML file and its corresponding mzML file in the same working directory. 


\section{Material S2, Trans-Proteomic Pipeline Tutorial:}

SPACEPro can be run from the Petunia web interface of the Trans-Proteomic Pipeline (TPP). This tutorial illustrates how to use SPACEPro following the database search and validation stages of a shotgun analysis pipeline. It is assumed the user knows and has generated their PeptideProphet or iProphet validated pepXML file from their database search results. For more information about how to operate the TPP, visit: http://www.tppms.org/

\section{Step 1:}

Select SPACEPro from the TPP TOOLS menu: TPP TOOLS->DIGESTION EFFICIENCY

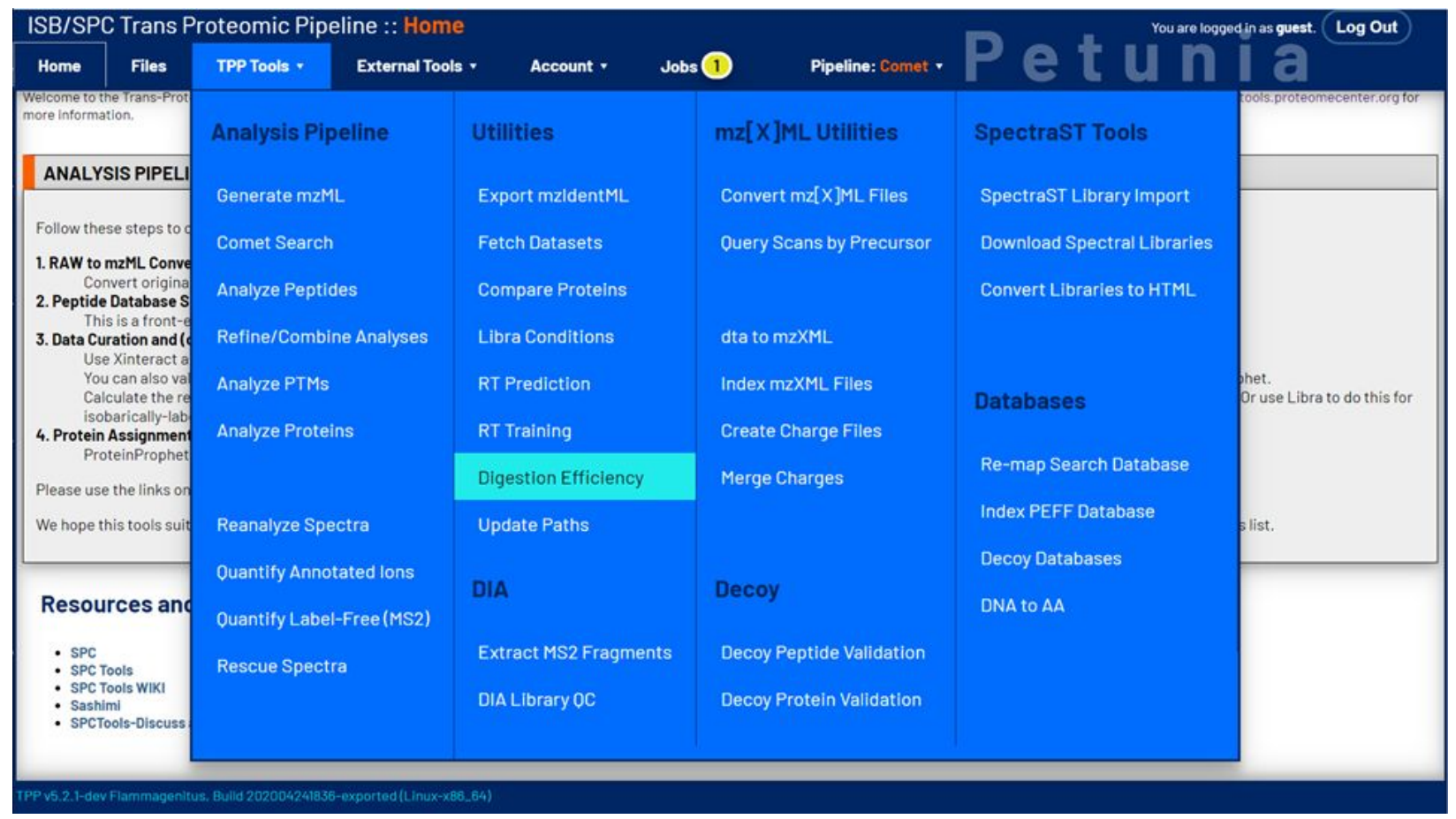




\section{Step 2:}

Select your PeptideProphet or iProphet validated PepXML file(s), set your desired parameters, and press the "Run SPACEPro" button.

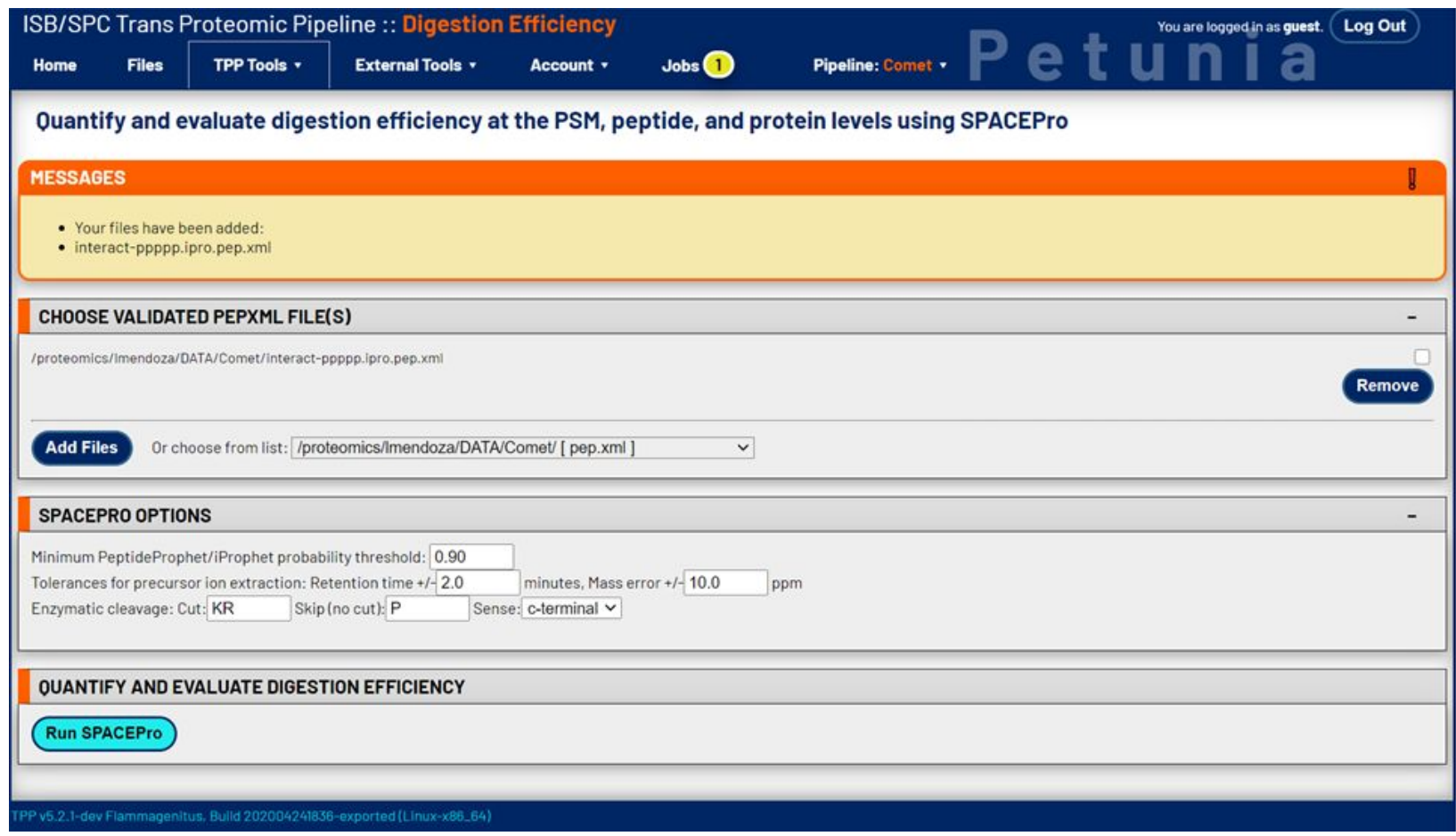




\section{Step 3:}

After completion of SPACEPro analysis, two output files are generated and reported. They are both text based formats (txt and JSON), and can be accessed from the file paths shown, for visualization or parsing with the user's tool of choice.

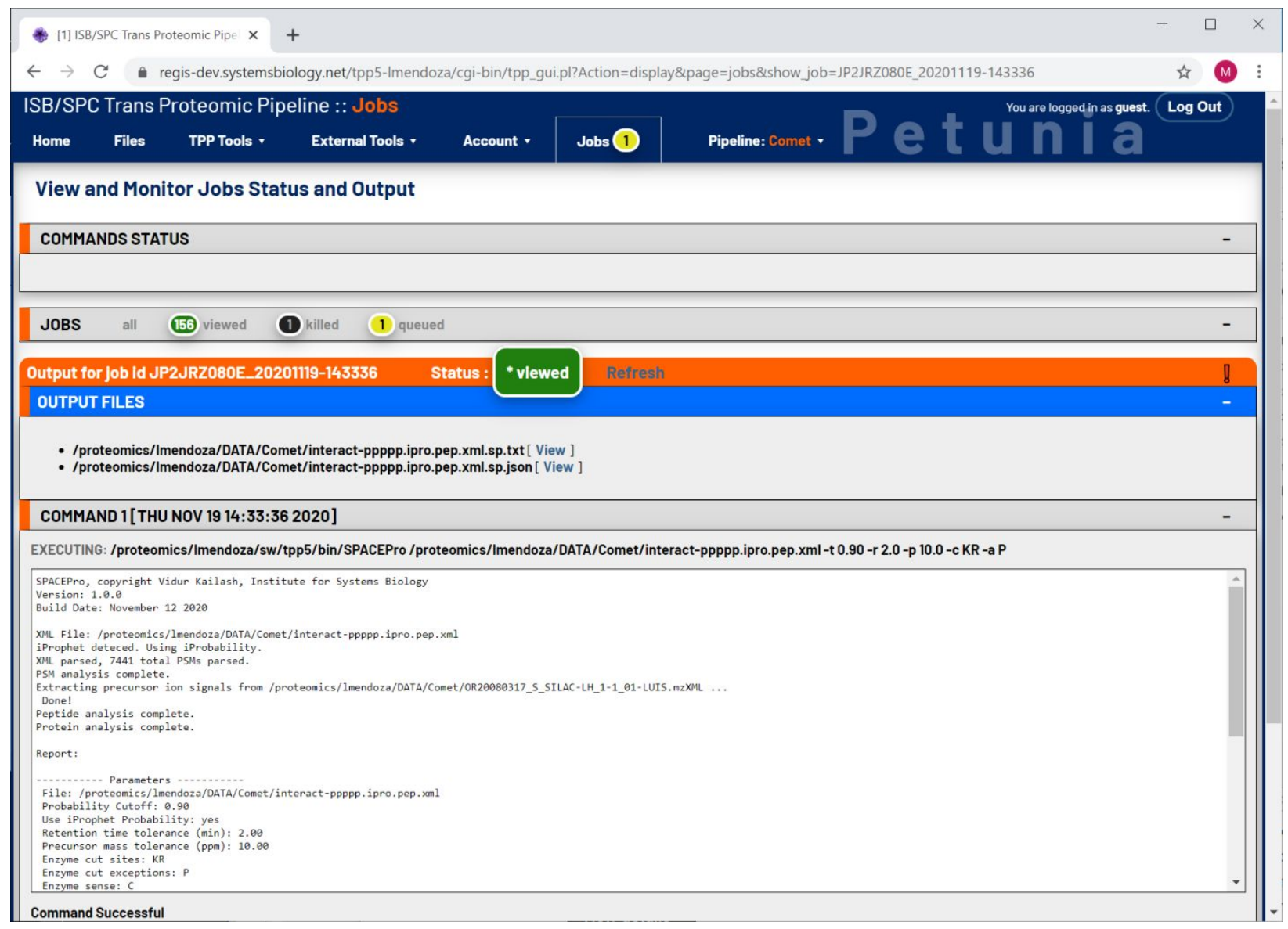

